\title{
Homotopy invariants of covers and KKM-type lemmas
}

\author{
OLEG R Musin
}

\begin{abstract}
Given any (open or closed) cover of a space $T$, we associate certain homotopy classes of maps from $T$ to $n$-spheres. These homotopy invariants can then be considered as obstructions for extending covers of a subspace $A \subset X$ to a cover of all of $X$. We use these obstructions to obtain generalizations of the classic KKM (KnasterKuratowski-Mazurkiewicz) and Sperner lemmas. In particular, we show that in the case when $A$ is a $k$-sphere and $X$ is a $(k+1)$-disk there exist KKM-type lemmas for covers by $n+2$ sets if and only if the homotopy group $\pi_{k}\left(\mathbb{S}^{n}\right)$ is nontrivial.
\end{abstract}

55M20, 55M25; 55P05

Throughout this paper we will consider only normal topological spaces, all simplicial complexes will be finite, all manifolds will be both compact and PL, $\mathbb{S}^{n}$ will denote the $n$-dimensional unit sphere, and $\mathbb{B}^{n}$ will denote the $n$-dimensional unit disk. We shall denote the set of homotopy classes of continuous maps from $X$ to $Y$ by $[X, Y]$.

\section{Homotopy invariants of covers}

First we consider labelings (colorings) of simplicial complexes. Denote by $\operatorname{Vert}(K)$ the vertex set of a simplicial complex $K$. (It is also referred to as the 0 -skeleton, $K^{0}$.) Let

$$
L: \operatorname{Vert}(K) \rightarrow\{0,1, \ldots, m\}
$$

be a labeling of the vertices of $K$. Denote by $\Delta^{m}$ an $m$-dimensional simplex with vertices $v_{0}, \ldots, v_{m}$. Let

$$
f_{L}(u):=v_{\ell}, \quad \text { where } u \in \operatorname{Vert}(K) \text { and } \ell=L(u) .
$$

Since $f_{L}$ is defined for all of the vertices of $K$, it induces a simplicial mapping $f_{L}:|K| \rightarrow\left|\Delta^{m}\right|$. This map is unique up to homeomorphism.

Note that if any simplex in $K$ has at most $m$ distinct labels, then $f_{L}$ is a map from $|K|$ to $\partial\left|\Delta^{m}\right| \cong \mathbb{S}^{m-1}$.

Definition 1.1 For a simplicial complex $K$ and a labeling $L$ : Vert $(K) \rightarrow\{0,1, \ldots, m\}$ such that $K$ has no simplices with $m+1$ distinct labels, we denote by $[L]$ the homotopy class $\left[f_{L}\right]$ in $\left[|K|, \mathbb{S}^{m-1}\right]$. 
Example 1.2 Let $K$ be a triangulation of $\mathbb{S}^{k}$ and $L: \operatorname{Vert}(K) \rightarrow\{0,1, \ldots, m\}$ be a labeling such that $K$ has no simplices with $m+1$ distinct labels. Then $[L] \in \pi_{k}\left(\mathbb{S}^{m-1}\right)$. In the case $k=m-1$ we have $\pi_{k}\left(\mathbb{S}^{m-1}\right)=\mathbb{Z}$ and

$$
[L]=\operatorname{deg}\left(f_{L}\right) \in \mathbb{Z} .
$$

(Here by $\operatorname{deg}(f)$ we denote the degree of a continuous map $f$ from $\mathbb{S}^{n}$ to itself.)

For instance, let $L$ be a Sperner labeling of a triangulation $K$ of $\partial \Delta^{m}=u_{0} u_{1} \cdots u_{m}$. The rules of this labeling are:

(i) The vertices of $\Delta^{m}$ are colored with different colors, ie $L\left(u_{i}\right)=i$ for $0 \leq i \leq m$.

(ii) Vertices of $K$ located on any $n$-dimensional subface of the large simplex $u_{i_{0}} u_{i_{1}} \cdots u_{i_{n}}$ are colored only with the colors $i_{0}, i_{1}, \ldots, i_{n}$.

Then $[L]=\operatorname{deg}\left(f_{L}\right)=1$ in $\left[\mathbb{S}^{m-1}, \mathbb{S}^{m-1}\right]=\mathbb{Z}$.

Example 1.3 Madahar and Sarkaria [7] considered a simplicial map $\tau: \mathbb{S}_{12}^{3} \rightarrow \mathbb{S}_{4}^{2}$ from a 12 -vertex 3 -sphere $\mathbb{S}_{12}^{3}$ onto the 4 -vertex 2 -sphere $\mathbb{S}_{4}^{2}$ (tetrahedron) with vertices $v_{0} v_{1} v_{2} v_{3}$. Actually, $\tau$ is a vertex-minimal simplicial map of Hopf invariant one.

For $u \in \operatorname{Vert}\left(\mathbb{S}_{12}^{3}\right)$, let $L_{\tau}(u):=i$, where $\tau(u)=v_{i}$. Then $\left[L_{\tau}\right]=1 \in \pi_{3}\left(\mathbb{S}^{2}\right)=\mathbb{Z}$.

Let $K$ be a simplicial complex. Denote by $\operatorname{St}(u)$ the open star of a vertex $u \in \operatorname{Vert}(K)$. In other words, $\operatorname{St}(u)$ is $|S| \backslash|B|$, where $S$ is the set of all simplices in $K$ that contain $u$, and $B$ is the set of all simplices in $S$ that do not contain $u$.

Let

$$
L: \operatorname{Vert}(K) \rightarrow\{0,1, \ldots, m\}
$$

be a labeling of the vertices of $K$. There is a natural open cover of $|K|$,

$$
\mathcal{U}_{L}(K)=\left\{U_{0}(K), \ldots, U_{m}(K)\right\},
$$

where

$$
U_{\ell}(K):=\bigcup_{u \in W_{\ell}} \operatorname{St}(u) \quad \text { and } \quad W_{\ell}:=\{u \in \operatorname{Vert}(K): L(u)=\ell\} .
$$

So with any labeling $L$ we associate a cover $\mathcal{U}_{L}(K)$. Now we extend Definition 1.1 to covers.

Let $\mathcal{U}=\left\{U_{0}, \ldots, U_{m}\right\}$ be an open finite cover of a space $T$. If $N(\mathcal{U})$ is its nerve, then there is a one-to-one correspondence between canonical maps $c: T \rightarrow|N(\mathcal{U})|$ and 
partitions of unity $\Phi$ subordinate to $\mathcal{U}$. Moreover, any two canonical maps $T \rightarrow|N(\mathcal{U})|$ are homotopic.

Since the nerve $N(\mathcal{U})$ is a subcomplex of the simplex $\Delta^{m}$, we have an embedding $\alpha:|N(\mathcal{U})| \rightarrow\left|\Delta^{m}\right|$. In the case when the intersection of all of the $U_{i}$ is empty, ie when $N(\mathcal{U})$ does not contain an $m$-cell, we have

$$
\alpha:|N(\mathcal{U})| \rightarrow \partial\left|\Delta^{m}\right| \cong \mathbb{S}^{m-1} .
$$

If

$$
\rho_{\mathcal{U}, c}:=\alpha \circ c,
$$

then a homotopy class $\left[\rho_{\mathcal{U}, c}\right]$ in $\left[T, \mathbb{S}^{m-1}\right]$ does not depend on the canonical map $c: T \rightarrow|N(\mathcal{U})|$.

Definition 1.4 Let $\mathcal{U}=\left\{U_{0}, \ldots, U_{m}\right\}$ be an open finite cover of a space $T$ such that the intersection of all of the $U_{i}$ is empty. Denote by $[\mathcal{U}]$ the homotopy class $\left[\rho_{\mathcal{U}, c}\right]$ in $\left[T, \mathbb{S}^{m-1}\right]$.

Remark It is clear that

$$
\left[\mathcal{U}_{L}(K)\right]=[L] \quad \text { in }\left[|K|, \mathbb{S}^{m-1}\right] .
$$

Theorem 1.5 Let $T$ be a space and $h$ be a homotopy class in $\left[T, \mathbb{S}^{m-1}\right]$. Then there is an open cover $\mathcal{U}=\left\{U_{0}, \ldots, U_{m}\right\}$ such that $[\mathcal{U}]=h$.

If $T$ is a simplicial complex, then there is a triangulation $K$ of $T$ and a labeling $L: \operatorname{Vert}(K) \rightarrow\{0,1, \ldots, m\}$ with $\left[\mathcal{U}_{L}(K)\right]=h$.

Proof Let $\Lambda: \operatorname{Vert}\left(\Delta^{m}\right) \rightarrow\{0,1, \ldots, m\}$ be a labeling of $\Delta^{m}$ with vertices $v_{0}, \ldots, v_{m}$ such that $\Lambda\left(v_{\ell}\right)=\ell$ for all $\ell$. Then we have a cover $\mathcal{U}_{\Lambda}\left(\Delta^{m}\right)$.

Let $f: T \rightarrow \mathbb{S}^{m-1}$ be a continuous map with $[f]=h$ and

$$
U_{\ell}:=f^{-1}\left(U_{\ell}\left(\Delta^{m}\right)\right) \text { for } \ell=0, \ldots, m \text {. }
$$

It is easy to see that $[\mathcal{U}]=h$.

If $T$ is a simplicial complex, then by the simplicial approximation theorem there is a simplicial subdivision (triangulation) $K$ and a simplicial map $g: K \rightarrow \Delta^{m}$ such that $g$ is homotopic to $f$. For all $v \in \operatorname{Vert}(K)$, let

$$
L(v):=\Lambda(g(v)) .
$$

Then $\left[\mathcal{U}_{L}(K)\right]=h$. 
Let us define the class $[\mathcal{U}]$ more explicitly. Let $\Phi=\left\{\varphi_{0}, \ldots, \varphi_{m}\right\}$ be a partition of unity subordinate to $\mathcal{U}$, and for all $x \in T$,

$$
\rho_{\mathcal{U}, \Phi}(x):=\sum_{i=0}^{m} \varphi_{i}(x) v_{i},
$$

where $v_{0}, \ldots, v_{m}$ are vertices of an $m$-dimensional simplex $V$ considered as vectors in $\mathbb{R}^{m}$. Then $\rho_{\mathcal{U}, \Phi}$ is a continuous map from $T$ to $\partial V=\mathbb{S}^{m-1}$. It is clear that

$$
\left[\rho_{\mathcal{U}, \Phi}\right]=[\mathcal{U}] \quad \text { in }\left[T, \mathbb{S}^{m-1}\right]
$$

Now we extend this definition. Let $V:=\left\{v_{0}, \ldots, v_{m}\right\}$ be any set of points (vectors) in $\mathbb{R}^{n+1}$. As above,

$$
\rho_{\mathcal{U}, \Phi, V}(x):=\sum_{i=0}^{m} \varphi_{i}(x) v_{i} .
$$

Suppose a point $p \in \mathbb{R}^{n+1}$ lies outside of the image $\rho_{\mathcal{U}, \Phi, V}(T)$. For all $x \in T$, let

$$
f_{\mathcal{U}, \Phi, V, p}(x):=\frac{\rho_{\mathcal{U}, \Phi, V}(x)-p}{\left\|\rho_{\mathcal{U}, \Phi, V}(x)-p\right\|} .
$$

Then $f_{\mathcal{U}, \Phi, V, p}$ is a continuous map from $T$ to $\mathbb{S}^{n}$.

Lemma 1.6 For given $\mathcal{U}, V$ and $p$, any two partitions of unity subordinate to $\mathcal{U}$ define the same homotopy class $\left[f_{\mathcal{U}, V, p}\right]$ in $\left[T, \mathbb{S}^{n}\right]$.

Proof A linear homotopy $\Theta(t)=(1-t) \Phi+t \Psi$ of two partitions of unity $\Phi$ and $\Psi$ induces a homotopy between the maps $f_{\mathcal{U}, \Phi, V, p}$ and $f_{\mathcal{U}, \Psi, V, p}$.

Lemma 1.7 For any two partitions of unity $\Phi$ and $\Psi$ subordinate to $\mathcal{U}$, the image $\rho_{\mathcal{U}, \Phi, V}(T)$ coincides with the image $\rho_{\mathcal{U}, \Psi, V}(T)$ in $\mathbb{R}^{n+1}$.

Proof Consider the nerve $N(\mathcal{U})$ with vertices $U_{i}$. If we set $g\left(U_{i}\right):=v_{i}$, then we have a piecewise linear map $g:|N(\mathcal{U})| \rightarrow H$, where $H:=\operatorname{conv}(V)$ is the convex hull of $V$ in $\mathbb{R}^{n+1}$. Then for any partition of unity $\Phi$, we have $\rho_{\mathcal{U}, \Phi, V}:=g \circ c$, where $c$ is the canonical map $c: T \rightarrow|N(\mathcal{U})|$ corresponding to $\Phi$. Thus, $\rho_{\mathcal{U}, \Phi, V}(T)$ equals $g(|N(\mathcal{U})|)$ and does not depend on $\Phi$.

Notation $P_{\mathcal{U}, V}(T):=\mathbb{R}^{n+1} \backslash \rho_{\mathcal{U}, \Phi, V}(T)$.

Note that the map $f_{\mathcal{U}, \Phi, V, p}: T \rightarrow \mathbb{S}^{n}$ is well defined only if $p \in P_{\mathcal{U}, V}(T)$. 
Lemma 1.8 Let points $p$ and $q$ lie in the same connected component $Q$ of $P_{\mathcal{U}, V}(T)$. Then $\left[f_{\mathcal{U}, V, p}\right]=\left[f_{\mathcal{U}, V, q}\right]$ in $\left[T, \mathbb{S}^{n}\right]$.

Proof Let $s(t)$ be a path in $Q$ connecting the points $p$ and $q$. Then $s$ induces a homotopy between the maps $f_{\mathcal{U}, \Phi, V, p}$ and $f_{\mathcal{U}, \Phi, V, q}$.

Definition 1.9 For a cover $\mathcal{U}=\left\{U_{1}, \ldots, U_{m}\right\}$ of a space $T$, a set $V$ of $m$ points in $\mathbb{R}^{n+1}$, and $p \in P_{\mathcal{U}, V}(T)$, denote the homotopy class $\left[f_{\mathcal{U}, V, p}\right]$ in $\left[T, \mathbb{S}^{n}\right]$ by $h(\mathcal{U}, V, p)$. For a labeling $L: \operatorname{Vert}(K) \rightarrow\{0,1, \ldots, m\}$ of a simplicial complex $K$ we denote by $h(K, L, V, p)$ the homotopy class $h\left(\mathcal{U}_{L}(K), V, p\right)$ in $\left[|K|, \mathbb{S}^{n}\right]$.

Example 1.10 Let $K$ be a heptagon with seven consecutive vertices labeled as $0,1,2,3,2,1,3$. Let $V=\left\{v_{0}, v_{1}, v_{2}, v_{3}\right\}$ be the set of vertices of a planar square. Then $h(K, L, V, p)=1$ if $p$ lies in the triangle $v_{0} v_{1} v_{3}$ and $h(K, L, V, p)=0$ otherwise.

Now we consider homotopy classes of covers of closed sets. Let $\mathcal{C}=\left\{C_{0}, \ldots, C_{m}\right\}$ be a closed cover of a space $T$. Let $\mathcal{U}=\left\{U_{0}, \ldots, U_{m}\right\}$ be an open cover of $T$ such that $U_{i}$ contains $C_{i}$ for all $i$. We say that $\mathcal{U}$ contains $\mathcal{C}$.

We may assume that the nerves $N(\mathcal{U})$ and $N(\mathcal{C})$ are isomorphic. Otherwise, if there is a subset of indices $J \subset\{0, \ldots, m\}$ such that the intersection of those $U_{i}$ whose subindices are in $J$ is nonempty and the intersection of those $C_{i}$ whose subindices are in $J$ is empty, we consider an open cover $\mathcal{U}^{\prime}$ with

$$
U_{i}^{\prime}:=U_{i} \backslash K_{J} \quad \text { and } \quad K_{j}:=\bigcap_{j \in J} \bar{U}_{j} .
$$

Since $C_{i} \cap K_{J}=\varnothing$, we have that $U_{i}^{\prime}$ contains $C_{i}$.

Suppose two open covers $\mathcal{U}^{1}$ and $\mathcal{U}^{2}$ both contain $\mathcal{C}$ and that $N\left(\mathcal{U}^{1}\right), N\left(\mathcal{U}^{2}\right)$ and $N(\mathcal{C})$ are isomorphic. Then the cover $\mathcal{U}^{3}$ given by $U_{i}^{3}:=U_{i}^{1} \cap U_{i}^{2}$ also contains $\mathcal{C}$. Moreover, $N\left(\mathcal{U}^{3}\right)$ is isomorphic to $N(\mathcal{C})$. Since both $\mathcal{U}^{1}$ and $\mathcal{U}^{2}$ contain $\mathcal{U}^{3}$, we have the equalities $h\left(\mathcal{U}^{1}, V, p\right)=h\left(\mathcal{U}^{3}, V, p\right)=h\left(\mathcal{U}^{2}, V, p\right)$.

This observation proves the following statement.

Lemma 1.11 Let $\mathcal{C}$ be a closed cover of a normal space $T$. Then there exists an open cover $\mathcal{U}$ of $T$ which contains $\mathcal{C}$ such that the nerves $N(\mathcal{U})$ and $N(\mathcal{C})$ are isomorphic. If open covers $\mathcal{U}^{1}$ and $\mathcal{U}^{2}$ both contain $\mathcal{C}$ and the nerves $N\left(\mathcal{U}^{1}\right), N\left(\mathcal{U}^{2}\right)$ and $N(\mathcal{C})$ are isomorphic, then $h\left(\mathcal{U}^{1}, V, p\right)=h\left(\mathcal{U}^{2}, V, p\right)$.

This lemma shows that the homotopy class $h(\mathcal{C}, V, p):=h(\mathcal{U}, V, p)$ in $\left[T, \mathbb{S}^{n}\right]$, where $N(\mathcal{U})=N(\mathcal{C})$ and $\mathcal{U}$ contains $\mathcal{C}$, is well defined. 


\section{Extension of covers}

In this section we consider extensions of covers of a subspace $A$ to a space $X$.

We call a family of sets a cover of a space if it is either an open or closed cover.

Definition 2.1 Let $A$ be a subspace of a space $X$. Let $\mathcal{S}=\left\{S_{0}, \ldots, S_{m}\right\}$ be a cover of $A$ and $\mathcal{F}=\left\{F_{0}, \ldots, F_{m}\right\}$ be a cover of $X$. We assume that $\mathcal{F}$ is open if $\mathcal{S}$ is open and closed if $\mathcal{S}$ is closed. We say that $\mathcal{F}$ is an extension of $\mathcal{S}$ if

$$
S_{i}=F_{i} \cap A \text { for all } i .
$$

We start from the classic case: $A=\mathbb{S}^{k}$ and $X=\mathbb{B}^{k+1}$.

Theorem 2.2 Let $\mathcal{S}=\left\{S_{0}, \ldots, S_{n+1}\right\}$ be a cover of $\mathbb{S}^{k}$. Suppose the intersection of all the $S_{i}$ is empty. Then $\mathcal{S}$ can be extended to a cover $\mathcal{F}$ of $\mathbb{B}^{k+1}$ such that the intersection of all the $F_{i}$ is empty if and only if $[\mathcal{S}]=0$ in $\pi_{k}\left(\mathbb{S}^{n}\right)$.

Proof If $\mathcal{S}$ can be extended to $\mathcal{F}$, then we have $\rho_{\mathcal{S}}: \mathbb{S}^{k} \rightarrow \mathbb{S}^{n}$ and $\rho_{\mathcal{F}}: \mathbb{B}^{k+1} \rightarrow \mathbb{S}^{n}$. Since $\rho_{\mathcal{S}}=\rho_{\mathcal{F}} \circ l$ and $l: \mathbb{S}^{k} \rightarrow \mathbb{B}^{k+1}$ is null-homotopic, we have $[\mathcal{S}]=\left[\rho_{\mathcal{S}}\right]=0$.

If $[\mathcal{S}]=0$, then we will show that $\mathcal{S}$ can be extended to a cover $\mathcal{F}$. From Lemma 1.11 it suffices to prove the theorem for open covers. Let $\Phi=\left\{\varphi_{0}, \ldots, \varphi_{n+1}\right\}$ be a partition of unity subordinate to $\mathcal{S}$. Then we have a continuous map

$$
\rho_{\mathcal{S}, \Phi}: \mathbb{S}^{k} \rightarrow \partial \Delta^{n+1}=\mathbb{S}^{n},
$$

where $\rho_{\mathcal{S}, \Phi}:=\rho_{\mathcal{S}, \Phi, V}$ (see Section 1$)$ and $V$ is the set of vertices of an $(n+1)$-simplex $\Delta^{n+1}$.

Since $\left[\rho_{\mathcal{S}, \Phi}\right]=0$ in $\left[\mathbb{S}^{k}, \mathbb{S}^{n}\right]$, there is a homotopy

$$
H: \mathbb{S}^{k} \times[0,1] \rightarrow \mathbb{S}^{n},
$$

where $H(x, 0)=\rho_{\mathcal{S}, \Phi}(x), H(x, 1)=v_{0}$ for all $x$, and $v_{0}$ is a vertex of $\Delta^{n+1}$.

Let $L$ be a labeling on $\operatorname{Vert}\left(\Delta^{n+1}\right)$ such that $L\left(v_{i}\right)=i$. Denote

$$
\left.U_{\ell}(\Phi, D):=H^{-1}\left(U_{\ell}\left(\Delta^{n+1}\right)\right)\right) \quad \text { and } \quad D:=\mathbb{S}^{k} \times[0,1]
$$

where $U_{L}\left(\Delta^{n+1}\right)=\left\{U_{\ell}\left(\Delta^{n+1}\right): \ell=1, \ldots, n+2\right\}$ (see Section 3). It is clear that $\mathcal{U}_{\ell}(\Phi, D):=\left\{U_{\ell}(\Phi, D)\right\}$ is an open cover of $D$, that $\mathcal{U}\left(\Phi, \mathbb{S}^{k}\right):=\left.\mathcal{U}(\Phi, D)\right|_{\mathbb{S}^{k}}$ is a cover of $\mathbb{S}^{k}$, and that

$$
U_{\ell}\left(\Phi, \mathbb{S}^{k}\right)=\left\{x \in \mathbb{S}^{k}: \varphi_{\ell}(x)>0\right\} \subset S_{\ell} \text { for all } \ell .
$$


Denote by $\Pi(\mathcal{S})$ the set of all partitions of unity subordinate to $\mathcal{S}$. Then for all $\ell$,

Let

$$
S_{\ell}=\bigcup_{\Phi \in \Pi(\mathcal{S})} U_{\ell}\left(\Phi, \mathbb{S}^{k}\right) \text {. }
$$

$$
W_{\ell}=\bigcup_{\Phi \in \Pi(\mathcal{S})} U_{\ell}(\Phi, D) .
$$

Then $\mathcal{W}:=\left\{W_{\ell}\right\}$ is an open cover of $D$ that extends $\mathcal{S}$.

The boundary of $D$ consists of two components $D_{0}:=\mathbb{S}^{k} \times\{0\}$ and $D_{1}:=\mathbb{S}^{k} \times\{1\}$. Actually, $\mathcal{W}$ on $D_{0}$ is $\mathcal{S}$ and $D_{1}$ is covered only by one set, namely $D_{1} \subset W_{0}$. Let $Z$ be a $(k+1)$-disk with boundary $D_{1}$ and let

$$
B:=D \cup Z, \quad \text { where } D \cap Z=D_{1} .
$$

It is clear that $B$ is homeomorphic to $\mathbb{B}^{k+1}$. Let $F_{0}:=W_{0} \cup Z$ in $B$ and let $\mathcal{F}:=$ $\left\{F_{0}, W_{1}, \ldots, W_{n+1}\right\}$. Then $\mathcal{F}$ is a cover of $B$ that extends $\mathcal{S}$.

Next consider the case when $A$ is the boundary of a manifold $X$.

Definition 2.3 Let $\mathcal{S}=\left\{S_{0}, \ldots, S_{n+1}\right\}$ be a cover of an oriented $n$-dimensional manifold $N$ without boundary. If the intersection of all the $S_{i}$ is empty, then

$$
[\mathcal{S}] \in \mathbb{Z}=\left[N, \mathbb{S}^{n}\right] .
$$

We call $[\mathcal{S}]$ the degree of $\mathcal{S}$ and denote it by $\operatorname{deg}(\mathcal{S})$.

Theorem 2.4 Let $M$ be an oriented $(n+1)$-dimensional manifold with boundary, and let $\mathcal{S}=\left\{S_{0}, \ldots, S_{n+1}\right\}$ be a cover of $\partial M$ such that the intersection of all the $S_{i}$ is empty. Then $\mathcal{S}$ can be extended to a cover $\mathcal{F}$ of $M$, such that all covers $F_{i}$ have no common point, if and only if the degree of $\mathcal{S}$ is zero.

Proof From the Hopf extension (degree) theorem it follows that a continuous map $f: \partial M \rightarrow \mathbb{S}^{n}$ can be extended to a globally defined continuous map $F: M \rightarrow \mathbb{S}^{n}$, with $\partial F=f$, if and only if the degree of $f$ is zero. This implies that if $\mathcal{S}$ can be extended, then $\operatorname{deg}\left(\rho_{\mathcal{S}}\right)=\operatorname{deg}(\mathcal{S})=0$.

If $\operatorname{deg}(\mathcal{S})=0$, then the proof that $\mathcal{S}$ can be extended is almost the same as the proof in Theorem 2.2. In the last step we can use, by the collar neighborhood theorem, that $\partial M$ has a neighborhood $C$ in $M$ which is homeomorphic to the product $D=\partial M \times[0,1]$. Let $F_{0}:=W_{0} \cup(M \backslash D)$. Then $\mathcal{F}:=\left\{F_{0}, W_{1}, \ldots, W_{n+1}\right\}$ is a cover of $M$ that extends $\mathcal{S}$. 
It is an interesting problem to find extensions of Theorems 2.2 and 2.4 for general $X, A$ and $V$.

For extensions of the KKM- and Sperner-type lemmas we need pairs of spaces $(X, A)$ such that covers of $A$ which are not null-homotopic cannot be extended to $X$. So we need only the "necessary" parts of Theorems 2.2 and 2.4. Note that pairs of spaces $(X, A)$ in these theorems satisfy the property that any continuous map $f: A \rightarrow \mathbb{S}^{n}$ with $[f] \neq 0$ cannot be extended to a continuous map $F: M \rightarrow \mathbb{S}^{n}$.

Let $\mathcal{S}=\left\{S_{0}, \ldots, S_{n+1}\right\}$ be a cover of $A$ and $\mathcal{F}=\left\{F_{0}, \ldots, F_{n+1}\right\}$ be a cover of $X$. Suppose that the intersection of all of the $S_{i}$ is empty. If $\mathcal{F}$ is an extension of $\mathcal{S}$ and the intersection of all of the $F_{i}$ is empty, then we have maps $\rho_{\mathcal{S}}: A \rightarrow \mathbb{S}^{n}$ and $\rho_{\mathcal{F}}: X \rightarrow \mathbb{S}^{n}$ such that $\left.\rho_{\mathcal{F}}\right|_{A}=\rho_{\mathcal{S}}$. This fact motivates the following definition.

Definition 2.5 We say that a pair of spaces $(X, A)$, where $A \subset X$, belongs to $\mathrm{EP}_{n}$ and write $(X, A) \in \mathrm{EP}_{n}$ if there is a continuous map $f: A \rightarrow \mathbb{S}^{n}$ with $[f] \neq 0$ in $\left[A, \mathbb{S}^{n}\right]$ that cannot be extended to a continuous map $F: X \rightarrow \mathbb{S}^{n}$ with $\left.F\right|_{A}=f$.

We denoted this class of pairs by EP after S Eilenberg and L S Pontryagin, who initiated obstruction theory in the late 1930s. Obstruction theory (see $[5 ; 15]$ ) considers homotopy invariants that equal zero if a map can be extended from the $k$-skeleton of $X$ to the $(k+1)$-skeleton and are nonzero otherwise.

We conclude this section with a theorem that is a simple consequence of obstruction theory.

Theorem 2.6 Let $(X, A)$ be a pair of spaces.

(1) If the embedding $\imath: A \rightarrow X$ is null-homotopic and there are non-null-homotopic maps $f: A \rightarrow \mathbb{S}^{n}$, then $(X, A) \in \mathrm{EP}_{n}$. In particular, if $\pi_{k}\left(\mathbb{S}^{n}\right) \neq 0$, then $\left(\mathbb{B}^{k+1}, \mathbb{S}^{k}\right) \in \mathrm{EP}_{n}$.

(2) If $X$ is an oriented $(n+1)$-dimensional manifold and $A=\partial X$, then $(X, A) \in \mathrm{EP}_{n}$.

Proof (1) Assume the conclusion is false. Then $f: A \rightarrow \mathbb{S}^{n}$, with $[f] \neq 0$, can be extended to $F: X \rightarrow \mathbb{S}^{n}$. Since $f=F \circ l$ and $[l]=0$ in $[A, X]$, we have that $[f]=0$ in $\left[A, \mathbb{S}^{n}\right]$, which is a contradiction.

(2) From the Hopf theorem $f: A \rightarrow \mathbb{S}^{n}$ can be extended if and only if $[f]=0$. 


\section{KKM- and Sperner-type lemmas}

The KKM (Knaster-Kuratowski-Mazurkiewicz) lemma states:

If a simplex $\Delta^{m}$ is covered by closed sets $C_{i}$ for $i \in I_{m}:=\{0, \ldots, m\}$ such that, for all $J \subset I_{m}$, the face of $\Delta^{m}$ that is spanned by the vertices $v_{i}$ with $i \in J$ is covered by $C_{i}$, then all the $C_{i}$ have a common intersection point.

This lemma was published in 1929 [6]. Actually, the KKM lemma is an extension of Sperner's lemma published one year before in 1928 [16].

Let $T$ be a triangulation of a simplex $\Delta^{m}$. Suppose that each vertex of $T$ is assigned a unique label from $I_{m}$. A labeling $L$ is called a Sperner labeling if the vertices are labeled in such a way that a vertex $u$ of $T$ belonging to a face that is spanned by vertices $v_{i}$ from $\operatorname{Vert}\left(\Delta^{m}\right)$ for $i \in J \subset I_{m}$ can only be labeled by $k$ from $J$. Sperner's lemma states:

Every Sperner labeling of a triangulation of $\Delta^{m}$ contains a cell labeled with a complete set of labels $\{0,1, \ldots, m\}$.

We consider extensions of the KKM and Sperner lemmas.

Theorem 3.1 Let $(X, A) \in \mathrm{EP}_{m-1}$ and let $\mathcal{S}=\left\{S_{0}, \ldots, S_{m}\right\}$ be a cover of $A$ such that the intersection of all the $S_{i}$ is empty and $[\mathcal{S}] \neq 0$ in $\left[A, \mathbb{S}^{m-1}\right]$. If $\mathcal{F}=\left\{F_{0}, \ldots, F_{m}\right\}$ is a cover of $X$ that extends $\mathcal{S}$, then all the $F_{i}$ have a common intersection point.

Proof Assume the conclusion is false. Then $\rho_{\mathcal{S}}: A \rightarrow \mathbb{S}^{m-1}$ can be extended to $\rho_{\mathcal{F}}: X \rightarrow \mathbb{S}^{m-1}$ which is a contradiction.

Theorem 2.6 implies that if $\pi_{k}\left(\mathbb{S}^{n}\right) \neq 0$, then $\left(\mathbb{B}^{k+1}, \mathbb{S}^{k}\right) \in \mathrm{EP}_{n}$.

Corollary 3.2 Let $\mathcal{F}=\left\{F_{0}, \ldots, F_{m}\right\}$ be a cover of $\mathbb{B}^{k+1}$ that extends a cover $\mathcal{S}$ of $\partial \mathbb{B}^{k+1}=\mathbb{S}^{k}$. If the intersection of all the $S_{i}$ is empty and $[\mathcal{S}] \neq 0$ in $\pi_{k}\left(\mathbb{S}^{m-1}\right)$, then all the $F_{i}$ have a common intersection point.

Note that for $k=m-1$ we have the KKM lemma. Indeed, the assumptions in this lemma yield that $[\mathcal{S}]=\operatorname{deg}(\mathcal{S})=1 \in \pi_{k}\left(\mathbb{S}^{m-1}\right)=\mathbb{Z}$.

It is interesting that this corollary can be nontrivial for $k>m-1$. Consider the cover $\mathcal{U}:=\mathcal{U}_{L_{\tau}}(K)=\left\{U_{0}(K), U_{1}(K), U_{2}(K), U_{3}(K)\right\}$, where $K=\mathbb{S}_{12}^{3}$, from Example 1.3. Since $[\mathcal{U}]=1 \in \pi_{3}\left(\mathbb{S}^{2}\right)=\mathbb{Z}$, Corollary 3.2 implies that: 
If a cover $\mathcal{F}=\left\{F_{0}, F_{1}, F_{2}, F_{3}\right\}$ of $\mathbb{B}^{4}$ is such that $\left.\mathcal{F}\right|_{\partial \mathbb{B}^{4}}=\mathcal{U}$, then the intersection of all the $F_{i}$ is not empty.

However, for $k=m=2$ any cover $\mathcal{S}=\left\{S_{0}, S_{1}, S_{2}\right\}$ of $\mathbb{S}^{2}$, where the $S_{i}$ have no common point, can be extended to a cover $\mathcal{F}$ of $\mathbb{B}^{3}$ such that the intersection of all the $F_{i}$ is empty. Actually, it follows from the fact that $\pi_{2}\left(\mathbb{S}^{1}\right)=0$.

Theorems 1.5 and 2.2 imply a condition for the existence of KKM-type lemmas for arbitrary positive integers $k$ and $m$.

Corollary 3.3 For given $k$ and $m$ there is a cover $\mathcal{S}=\left\{S_{0}, \ldots, S_{m}\right\}$ of $\mathbb{S}^{k}$ such that the intersection of all the $S_{i}$ is empty, and for any cover $\mathcal{F}$ of $\mathbb{B}^{k+1}$ that extends $\mathcal{S}$, all the $F_{i}$ have a common intersection point if and only if $\pi_{k}\left(\mathbb{S}^{m-1}\right) \neq 0$.

Now we extend Theorem 3.1 for homotopy classes $h(\mathcal{S}, V, p)$.

Definition 3.4 Let $V:=\left\{v_{0}, \ldots, v_{m}\right\}$ be a set of points in $\mathbb{R}^{d}$. Consider a point $p \in \mathbb{R}^{d}$. Denote by $\operatorname{cov}_{V}(p)$ the collection of all subsets $J$ in $I_{m}$ such that simplices (convex hulls) in $\mathbb{R}^{d}$ spanned by vertices $\left\{v_{j}: j \in J\right\}$ contain $p$.

It is clear that we have the following:

Proposition 3.5 Let $\mathcal{S}=\left\{S_{0}, \ldots, S_{m}\right\}$ be a cover of a space $T$. Let $V:=\left\{v_{0}, \ldots, v_{m}\right\}$ be a set of points in $\mathbb{R}^{d}$, and let $p \in \mathbb{R}^{d}$. Then $p \in P_{\mathcal{U}, V}(T)$ if and only if, for any $J \in \operatorname{cov}_{V}(p)$, the intersection of the $S_{i}$ whose subindices $i$ are in $J$ is empty.

Theorem 3.6 Let $(X, A) \in \mathrm{EP}_{n}$. Let $\mathcal{S}=\left\{S_{0}, \ldots, S_{m}\right\}$ and $\mathcal{F}=\left\{F_{0}, \ldots, F_{m}\right\}$ be covers of $A$ and $X$, respectively. Let $V:=\left\{v_{0}, \ldots, v_{m}\right\}$ be a set of points in $\mathbb{R}^{n+1}$, and let $p \in \mathbb{R}^{n+1}$. Suppose $\mathcal{F}$ extends $\mathcal{S}$, for all $J \in \operatorname{cov}_{V}(p)$ the intersection of the $S_{j}$ whose subindices are in $J$ is empty, and

$$
h(\mathcal{S}, V, p) \neq 0 \quad \text { in }\left[A, \mathbb{S}^{n}\right] .
$$

Then there is a $J \in \operatorname{cov}_{V}(p)$ such that

$$
\bigcap_{j \in J} F_{j} \neq \varnothing
$$

Proof Assume the conclusion is false. Then $p \in \mathbb{R}^{n+1} \backslash \rho_{\mathcal{F}, V}(X)$. Therefore, $f_{\mathcal{F}, V, p}: X \rightarrow \mathbb{S}^{n}$ is well defined. On the other hand, it is an extension of the map $f_{\mathcal{S}, V, p}: A \rightarrow \mathbb{S}^{n}$ with $\left[f_{\mathcal{S}, V, p}\right] \neq 0$, which is a contradiction. 
Theorem 3.6 implies a generalization of Sperner's lemma:

Theorem 3.7 Let $X=|K|$ and $A=|Q|$, where $K$ is a simplicial complex and $Q$ is a subcomplex of $K$. Suppose $(X, A) \in \mathrm{EP}_{n}$. Let $L: \operatorname{Vert}(K) \rightarrow\{0,1, \ldots, m\}$ be a labeling of $K$. Let $V:=\left\{v_{0}, \ldots, v_{m}\right\}$ be a set of points in $\mathbb{R}^{n+1}$, and let $p \in \mathbb{R}^{n+1}$. Suppose there are no simplices in $Q$ whose vertices are labeled by a $J \in \operatorname{cov}_{V}(p)$. Let

$$
h(Q, L, V, p) \neq 0 \quad \text { in }\left[|Q|, \mathbb{S}^{n}\right] .
$$

Then there is a simplex $s$ in $K$ and there is a $J \in \operatorname{cov}_{V}(p)$ such that vertices of $s$ have labels $J$.

If $m=n+1$ and $[L] \neq 0$ in $\left[|Q|, \mathbb{S}^{n}\right]$, then there is a simplex in $K$ that has all the labels $0, \ldots, n+1$.

There are many generalizations of the KKM and Sperner lemmas; see $[1 ; 3 ; 4 ; 8 ; 10$; $11 ; 12 ; 13 ; 14 ; 17]$. Some of them follow from Theorems 3.6 and 3.7. For example, we consider here an extension of the Tucker-Bacon lemma (see [1; 17] and [14]).

Corollary 3.8 Let $(X, A) \in \mathrm{EP}_{n}$. Let $\mathcal{F}=\left\{F_{1}, F_{-1}, \ldots, F_{n}, F_{-n}\right\}$ be a cover of $X$ that extends a cover $\mathcal{S}$ of $A$. Suppose $S_{i} \cap S_{-i}=\varnothing$ for all $i$ and $h(\mathcal{S}, V, O) \neq 0$ in $\left[A, \mathbb{S}^{n-1}\right]$, where $V:=\left\{ \pm e_{1}, \ldots, \pm e_{n}\right\}$, with $e_{1}, \ldots, e_{n}$ an orthonormal basis and $O$ the origin in $\mathbb{R}^{n}$. Then there is an $i$ such that the intersection of $F_{i}$ and $F_{-i}$ is not empty.

Proof Note that $\operatorname{cov}_{V}(O)$ consists of edges that join $e_{i}$ and $\left(-e_{i}\right)$. Then Theorem 3.6 yields a proof.

Consider the case when $X=M$ is an oriented manifold of dimension $n+1$ and $A=\partial M$. Then $\left[A, \mathbb{S}^{n}\right]=\mathbb{Z}$ and for any continuous $f: A \rightarrow \mathbb{S}^{n}$ we have $[f]=\operatorname{deg} f$. Now we show that we can improve Theorem 3.1 in this case.

Let $s$ be a $d$-simplex. We say that $s$ is fully labeled (or colored) if vertices of $s$ are labeled (colored) by distinct labels $\ell_{0}, \ldots, \ell_{d}$.

Let $T$ be a triangulation of $M$. Let $L: \operatorname{Vert}(M) \rightarrow\{0,1, \ldots, n+1\}$ be a labeling of vertices. Let $\partial T$ denote the triangulation $T$ on $\partial M$. We denote by $\operatorname{deg}(L, \partial T)$ the class $[\partial T, L]$ in $\left[\partial M, \mathbb{S}^{n}\right]$.

Theorem 3.9 Let $T$ be a triangulation of a manifold $M$ of dimension $n$ with boundary. Then for a labeling $L: \operatorname{Vert}(T) \rightarrow\{0,1, \ldots, n\}$, the triangulation $T$ must contain at least $|\operatorname{deg}(L, \partial T)|$ fully colored simplices. 
Proof Actually, $L$ induces a piecewise linear map $f_{L}: T \rightarrow \Delta^{n}$, where $f_{L}=\rho_{\mathcal{U}_{L}}(T)$ and $\operatorname{deg} f_{L}=\operatorname{deg}(L, \partial T)$. Then any internal point $y$ in $\Delta^{n}$ is regular for $f_{L}$, the set of preimages $f_{L}^{-1}(y)$ consists of points $u_{k} \in M$ such that every $u_{k}$ lies inside some fully labeled $n$-simplex $t_{k} \in T$, and the sum of the signs of $u_{k}$ is equal to $\operatorname{deg} f_{L}$. This proves the theorem.

Let $P$ be a convex polytope in $\mathbb{R}^{d}$ with vertices $\left\{v_{1}, \ldots, v_{m}\right\}$. Let $T$ be a triangulation of a manifold $M$ of dimension $d$. Let $L: \operatorname{Vert}(T) \rightarrow\{1,2, \ldots, m\}$ be a labeling of $T$. If, for $u \in \operatorname{Vert}(T)$, we have $L(u)=i$, then we set $f_{L, P}(u):=v_{i}$. Therefore, $f_{L, P}$ is defined for all vertices of $T$, and it uniquely defines a simplicial (piecewise linear) map $f_{L, P}: M \rightarrow \mathbb{R}^{d}$.

The following theorem extends Theorems 3.7 and 3.9 and the De Loera-Petersen-Su theorem [3].

Theorem 3.10 Let $P$ be a convex polytope in $\mathbb{R}^{d}$ with $m$ vertices. Let $T$ be a triangulation of an oriented manifold $M$ of dimension $d$ with boundary. Let $L: \operatorname{Vert}(T) \rightarrow\{1,2, \ldots, m\}$ be a labeling such that $f_{L, P}(\partial M) \subseteq \partial P$. Then $T$ contains at least $(m-d)|\operatorname{deg}(L, \partial T)|$ fully labeled $d$-simplices.

Proof Consider a set of points $S$ in the interior of $P$ so that the interior of every simplex determined by $d+1$ vertices in $V:=\operatorname{Vert}(P)$ contains a unique point from $S$. In other words, for any two distinct points $x$ and $y$ in $S$, the intersection of the sets $\operatorname{cov}_{V}(x)$ and $\operatorname{cov}_{V}(y)$ is empty. Such sets have been called pebble sets by De Loera, Peterson, and Su. In [3], they proved that in $P$ there is a pebble set of cardinality at least $m-d$. Note that $\operatorname{deg}\left(f_{L, P}\right)=h(\partial T, L, V, p)$ for any internal point $p$ in $P$. Let us apply Theorem 3.7 for all points $p$ in $S$. Using the same argument about the number of preimages of $f_{L, P}^{-1}(p)$ as in Theorem 3.9, we prove the theorem.

We conclude this paper with an extension of Theorem 3.10 for simplicial complexes. Let $K$ be a $d$-dimensional simplicial complex. ED Bloch [2] defines the "boundary" of $K$, denoted Bd $K$, as the collection of all $(d-1)$-simplices of $K$ that are contained in an odd number of $d$-simplices, together with all the faces of these $(d-1)$-simplices.

Let $P$ be a convex polytope in $\mathbb{R}^{d}$ with $m$ vertices. Any labeling of the vertices of $K$, $L: \operatorname{Vert}(K) \rightarrow\{1,2, \ldots, m\}$, defines a simplicial map $f_{L, P}:|K| \rightarrow P \subset \mathbb{R}^{d}$. So we have a map $\left.f_{L, P}\right|_{|\mathrm{Bd} K|}: \operatorname{Bd}(X) \rightarrow \partial P \simeq \mathbb{S}^{d-1}$. Let us denote the degree of this map modulo 2 by $\operatorname{deg}_{2}(L, \mathrm{Bd} K)$. From [2, Theorem 1.5] it follows that the cardinality of $f_{L, P}^{-1}(p)$, where $p$ lies inside $P$, is equal to $\operatorname{deg}_{2}(L, \operatorname{Bd} K)$ modulo 2 . Then the pebble set theorem [3] yields the following theorem. 
Theorem 3.11 Let $P$ be a convex polytope in $\mathbb{R}^{d}$ with $m$ vertices. Let $T$ be a triangulation of a simplicial complex $X$ of dimension $d$. Let $L$ : $\operatorname{Vert}(T) \rightarrow\{1,2, \ldots, m\}$ be a labeling such that $f_{L, P}(|\mathrm{Bd} T|) \subseteq \partial P$. If $\operatorname{deg}_{2}(L, \mathrm{Bd} T)$ is odd, then $T$ contains at least $(m-d)$ fully labeled $d$-simplices.

Corollary 3.12 Let $T$ be a triangulation of a simplicial complex $X$ of dimension $d$. If $\operatorname{deg}_{2}(L, \operatorname{Bd} T)$ for a labeling $L: \operatorname{Vert}(T) \rightarrow\{1,2, \ldots, d+1\}$ is odd, then $T$ must contain at least one fully colored $d$-simplex.

The KKM lemma and its relatives have many applications in several fields of pure and applied mathematics. In [9] we consider some extensions of results of this paper that can be applied in game theory and mathematical economics.

Acknowledgements I wish to thank Alexander Dranishnikov, James Maissen and Alexey Volovikov for helpful discussions and comments. The research was carried out at the Institute for Information Transmission Problems, Russian Academy of Sciences, at the expense of the Russian Foundation for Sciences (project number 14-50-00150).

\section{References}

[1] P Bacon, Equivalent formulations of the Borsuk-Ulam theorem, Canad. J. Math. 18 (1966) 492-502 MR

[2] E D Bloch, Mod 2 degree and a generalized no retraction theorem, Math. Nachr. 279 (2006) 490-494 MR

[3] J A De Loera, E Peterson, F E Su, A polytopal generalization of Sperner's lemma, J. Combin. Theory Ser. A 100 (2002) 1-26 MR

[4] K Fan, A generalization of Tucker's combinatorial lemma with topological applications, Ann. of Math. 56 (1952) 431-437 MR

[5] S-t Hu, Homotopy theory, Pure and Applied Mathematics, Vol. VIII, Academic Press, New York-London (1959) MR

[6] B Knaster, C Kuratowski, S Mazurkiewicz, Ein Beweis des Fixpunktsatzes für ndimensionale Simplexe, Fundam. Math. 14 (1929) 132-137

[7] K V Madahar, K S Sarkaria, A minimal triangulation of the Hopf map and its application, Geom. Dedicata 82 (2000) 105-114 MR

[8] F Meunier, Sperner labellings: a combinatorial approach, J. Combin. Theory Ser. A 113 (2006) 1462-1475 MR

[9] O R Musin, KKM type theorems with boundary conditions, preprint arXiv 
[10] O R Musin, Borsuk-Ulam type theorems for manifolds, Proc. Amer. Math. Soc. 140 (2012) 2551-2560 MR

[11] O R Musin, Extensions of Sperner and Tucker's lemma for manifolds, J. Combin. Theory Ser. A 132 (2015) 172-187 MR

[12] O R Musin, Sperner type lemma for quadrangulations, Mosc. J. Comb. Number Theory 5 (2015) 26-35

[13] O R Musin, Generalizations of Tucker-Fan-Shashkin lemmas, preprint (2016) arXiv To appear in Arnold Math. J.

[14] O R Musin, A Y Volovikov, Borsuk-Ulam type spaces, Mosc. Math. J. 15 (2015) 749-766 MR

[15] E H Spanier, Algebraic topology, McGraw-Hill, New York (1966) MR

[16] E Sperner, Neuer beweis für die invarianz der dimensionszahl und des gebietes, Abh. Math. Sem. Univ. Hamburg 6 (1928) 265-272 MR

[17] A W Tucker, Some topological properties of disk and sphere, from: "Proc. First Canadian Math. Congress”, University of Toronto (1946) 285-309 MR

Department of Mathematics, University of Texas Rio Grande Valley

One West University Boulevard, Brownsville, TX 78520, United States

Institute for Information Transmission Problems, Russian Academy of Sciences Bolshoy Karetny per. 19, Moscow 127994, Russia

oleg.musin@utrgv.edu

Received: 21 June 2015 Revised: 7 September 2015 